\title{
The Effect of Nucleus Implant Parameters on the Compressive Mechanics of the Lumbar Intervertebral Disc: A Finite Element Study
}

\author{
Abhijeet Joshi, ${ }^{1}$ Christopher J. Massey, ${ }^{2}$ Andrew Karduna, ${ }^{3}$ Edward Vresilovic, ${ }^{4}$ Michele Marcolongo ${ }^{1}$ \\ ${ }^{1}$ Department of Materials Science and Engineering, Drexel University, Philadelphia, Pennsylvania \\ ${ }^{2}$ Department of Mechanical Engineering and Mechanics, Drexel University, Philadelphia, Pennsylvania \\ ${ }^{3}$ Department of Human Physiology, University of Oregon, Eugene, Oregon \\ ${ }^{4}$ Department of Orthopaedic Surgery, Pennsylvania State University, Hershey, Pennsylvania
}

Received 6 February 2008; revised 18 September 2008; accepted 24 October 2008

Published online 29 January 2009 in Wiley InterScience (www.interscience.wiley.com). DOI: 10.1002/jbm.b.31322

\begin{abstract}
A simplified finite element model of the human lumbar intervertebral disc was utilized for understanding nucleus pulposus implant mechanics. The model was used to assess the effect of nucleus implant parameter variations on the resulting compressive biomechanics of the lumbar anterior column unit. The effects of nucleus implant material (modulus and Poisson's ratio) and geometrical (height and diameter) parameters on the mechanical behavior of the disc were investigated. The model predicted that variations in implant modulus contribute less to the compressive disc mechanics compared to the implant geometrical parameters, for the ranges examined. It was concluded that some threshold exists for the nucleus implant modulus, below which little variations in load-displacement behavior were shown. Compressive biomechanics were highly affected by implant volume (under-filling the nucleus cavity, line-to-line fit, or over-filling the nucleus cavity) with a greater restoration of compressive mechanics observed with the over-filled implant design. This work indicated the effect of nucleus implant parameter variations on the compressive mechanics of the human lumbar intervertebral disc and importance of the "fit and fill" effect of the nuclear cavity in the restoration of the human intervertebral disc mechanics in compression. These findings may have clinical significance for nucleus implant design. (C) 2009 Wiley Periodicals, Inc. J Biomed Mater Res Part B: Appl Biomater 90B: 596-607, 2009
\end{abstract}

Keywords: finite element analysis; intervertebral disc; hydrogel; biomechanics; implant design

\section{INTRODUCTION}

Nucleus replacement by a polymeric material and by tissue engineering approaches are currently being investigated ${ }^{1-11}$ to treat chronic lower back pain. The motivation behind this approach is to use a minimally invasive technique to treat early degenerative discs as an alternative to the current invasive surgical procedures such as discectomy, spinal fusion, and total disc replacement, ${ }^{12-14}$ as well as the ability to intervene at an earlier stage of degeneration. Although these procedures can relieve back pain, they fail to restore the normal biomechanics of the lumbar spine.

Correspondence to: M. Marcolongo (e-mail: marcolms@drexel.edu)

Contract grant sponsor: NSF; contract grant number: BES 0085383

(C) 2009 Wiley Periodicals, Inc.
Discectomy may result in additional stress within the disc ${ }^{12}$ and instability. ${ }^{15}$ Spinal fusion may generate additional stress in adjacent discs after surgery, and the patient may lose mobility permanently. ${ }^{16,17}$ Total disc replacement may serve to eliminate pain while restoring physiological motion, ${ }^{18}$ however this technique is reserved for severely degenerated discs.

The intervertebral disc is the largest avascular tissue in the human body and is mainly composed of three different tissues. ${ }^{17}$ The central core, the nucleus pulposus, is surrounded by the outer annulus fibrosus and the upper and lower cartilaginous end plates. Lower back pain was reported in more than $80 \%$ of the cases exhibiting degeneration of lumbar intervertebral discs. ${ }^{19}$ With aging, the proteoglycan and water content in the central nucleus reduces significantly, ${ }^{20-24}$ causing abnormal loading to the outer annulus. ${ }^{17,25}$ In a dehydrated disc, the function of the 
nucleus, namely load transfer to the annulus through creation of an intradiscal pressure, is no longer occurring at a normal level. The mechanics of the degenerated disc are clearly altered compared to those of the intact disc. ${ }^{25,26}$

We propose the replacement of the degenerated nucleus using a polymeric hydrogel. ${ }^{27-30}$ Prior studies in our laboratory have focused on the development of a stable polymeric hydrogel to serve as a replacement to the degenerated nucleus pulposus. ${ }^{30,31}$ In our previous experimental studies, the effect of a hydrogel nucleus replacement on the compressive stiffness of the lumbar intervertebral disc was assessed using a human lumbar cadaveric model. In that work, the feasibility of replacing the nucleus with the hydrogel implant was demonstrated. A novel trans-end plate approach for in vitro testing of the nucleus implant was developed, to avoid injury to the annulus. ${ }^{3}$ This was achieved by creating a bone plug from the superior vertebra using a standard core drill. The cylindrical hydrogel implant restored $88 \%$ of the compressive stiffness of the intact disc when implanted in the created nuclear defect. ${ }^{3}$ In another experimental study, we assessed the effect of nucleus implant parameter variations (material and geometric) on the alteration of compressive stiffness of the lumbar disc and thus the compressive biomechanics of the disc. ${ }^{28}$ It was found that nucleus implant parameters do have a significant effect on the disc compressive mechanics. The cylindrical implant geometrical variations (height and diameter) were found to be more effective in restoring the disc compressive mechanics compared to the implant material (modulus) variations, in the range examined. Interestingly, a corollary of this result was that implant diameter was more effective in restoring the compressive stiffness than the implant height. Thus, a small increase in implant diameter (6\% press fit) achieved the stiffness level that was produced with $10 \%$ press fit of the implant height and $900 \%$ increase in the implant modulus. ${ }^{28}$ However, little could be determined from the experimental results regarding the stress state of the disc or the effect of undersized/ oversized implants (fit-fill effect of the nuclear cavity) on the overall mechanical behavior of the disc.

Previous work has been performed in the numerical modeling of lumbar intervertebral disc mechanics under various physiological conditions to better understand the role of the native disc tissues under different loading conditions. ${ }^{26,32-40}$ However, there is scant literature on the use of modeling to understand nucleus implant behavior. ${ }^{40,41}$ It was hypothesized by Bao and Yuan $^{42}$ that both nucleus implant modulus and nucleus implant cavity/conformity can affect the load distribution in the intervertebral disc. Meakin et al. used sheep discs to assess the effect of nucleus replacement by synthetic implant ${ }^{41}$; it was observed that the inner layers of the annulus bulged inwards in a denucleated disc. This inner bulging of the annulus was prevented by synthetic implants, when implanted in the denucleated disc. The experimental work with the sheep disc was further supported by a simplified finite element modeling of the intact human disc to investigate the effect of nucleus replacement. The model predicted that the effects of denucleation can be almost completely reversed by a solid implant having line-to-line fit with a Young's Modulus of $3 \mathrm{MPa}$. However, the nucleus implant and the annulus were modeled as elastic, isotropic materials, which precluded the consideration of material nonlinearity in the model. Yao et al. created a three-dimensional finite element model (FEM) to determine the ideal material properties of a tissue-engineered scaffold and which also accounted for the nonlinearities of the annulus. ${ }^{40}$ However, these values were compared to a model which described the nucleus as an incompressible fluid, while nucleus pulposus tissue becomes less fluid-like with age and degeneration. ${ }^{17}$

The objective of the present study was to determine the effect of nucleus implant parameters on the mechanical behavior of the lumbar intervertebral disc. The model we have proposed here is a next step to the model proposed by Meakin et al., ${ }^{41}$ in the sense that it accommodates the annulus material nonlinearity to have a better understanding of the nucleus replacement implant mechanics, while maintaining simplified axisymmetric geometry to maintain reasonable computing efforts.

\section{METHODS}

\section{Model Construction and Material Properties}

The properties of the cortical/cancellous bone of the vertebral body are well established and were taken from the literature. ${ }^{36}$ The literature is more varied for the experimentally reported nucleus and annulus material properties. ${ }^{35,39,41,43,44}$

The nucleus pulposus of each of six human cadaveric lumbar specimens displayed some degree of age-appropriate degeneration, and appeared more solid-like than fluid. For this reason, the nucleus pulposus of each specimen was modeled as an isotropic, elastic material with Young's modulus of $1 \mathrm{MPa}$ and Poisson's ratio (0.4999), in contrast with the previously reported method of modeling the nucleus as an incompressible fluid. ${ }^{39,40}$ Degenerated discs are often more fibrous than fluid-like, yet they still have the fluidic components, suggesting that the material behavior of the adult nucleus is better modeled as a linearly elastic solid with a high Poisson's ratio. The natural nucleus transfers axial loads to the annulus by creating an intradiscal pressure on the inner walls of the annulus and thereby displacing it in the outward radial direction. To simulate this natural load transfer phenomenon effectively, the highest possible Poisson's ratio was used for the nucleus. Poroelastic theory has been used to describe the nucleus and annulus, ${ }^{35,38,39}$ however, that approach was not used in this study due to the loading conditions (quasi-static compression vs. creep).

The complex anisotropic, nonlinear behavior of the annulus was simplified and the annulus was modeled as an 
TABLE I. Annulus Material Parameters for Second-Order Polynomial Strain Energy Function (all values in N/m²)

\begin{tabular}{|c|c|c|c|c|c|c|c|}
\hline & $C_{10}$ & $C_{01}$ & $C_{20}$ & $C_{11}$ & $C_{02}$ & $D_{1}$ & $D_{2}$ \\
\hline Specimen 1 & 3E04 & $8 \mathrm{E} 04$ & 3E04 & $3 \mathrm{E} 04$ & $5 \mathrm{E} 04$ & $1 \mathrm{E}-07$ & $1 \mathrm{E}-07$ \\
\hline Specimen 2 & $1 \mathrm{E} 05$ & $1 \mathrm{E} 05$ & $1 \mathrm{E} 05$ & $1 \mathrm{E} 05$ & $4 \mathrm{E} 05$ & $1 \mathrm{E}-07$ & $1 \mathrm{E}-07$ \\
\hline Specimen 3 & 4E04 & 4E04 & 4E05 & 4E05 & 4E05 & $1 \mathrm{E}-07$ & $1 \mathrm{E}-07$ \\
\hline Specimen 4 & 7E04 & 7E04 & 1E05 & 1E05 & 3E05 & $1 \mathrm{E}-07$ & $1 \mathrm{E}-07$ \\
\hline Specimen 5 & $8 \mathrm{E} 04$ & $8 \mathrm{E} 04$ & $2 \mathrm{E} 05$ & $2 \mathrm{E} 05$ & $3 \mathrm{E} 05$ & $1 \mathrm{E}-07$ & $1 \mathrm{E}-07$ \\
\hline Specimen 6 & $5 \mathrm{E} 04$ & $6 \mathrm{E} 04$ & 2E05 & 3E05 & $3 \mathrm{E} 05$ & $1 \mathrm{E}-07$ & $1 \mathrm{E}-07$ \\
\hline AVFEM & $6 \mathrm{E} 04$ & 7E04 & 2E05 & 2E05 & 3E05 & $1 \mathrm{E}-07$ & $1 \mathrm{E}-07$ \\
\hline
\end{tabular}

isotropic, hyperelastic material using a second-order polynomial strain energy function. To validate the FEMs, the load-displacement prediction from the individual models was matched to the physical testing results of the corresponding specimen by means of this strain energy function, which inherently accounts for the lack of fibers in the simulation. The definition of the annulus as an isotropic, hyperelastic material induced the required nonlinearity in the model to validate the simulation against the physical testing results. The definition of the annulus as an isotropic material is a reasonable assumption because only uniaxial compression loading was analyzed in this study. The true anisotropic nature of the annulus becomes relevant to mechanical behavior only in other loading modes such as torsion, bending, or coupled loading. The coefficients of the second-order polynomial function were determined by adjusting and matching the FEM predicted load-displacement curve with that of the intact experimental load-displacement curve, while keeping the material properties of the nucleus and vertebrae constant. ${ }^{3}$ Thus, each intact specimen was modeled with unique annulus parameters in the form of the polynomial strain energy potential:

$$
U=\sum_{i+j=1}^{N} C_{i j}\left(\bar{I}_{1}-3\right)^{i}\left(\bar{I}_{2}-3\right)^{j}+\sum_{i=1}^{N} \frac{1}{D_{i}}\left(J_{\mathrm{el}}-1\right)^{2 i}
$$

where $U$ is the strain energy potential, $C_{i j}$ and $D_{i}$ are material parameters (Table I), $J_{\mathrm{el}}$ is the elastic volume strain, and $I_{1}$ and $I_{2}$ are the first and second strain invariants, respectively. ${ }^{45}$ The same annulus parameters for each specimen were used in the corresponding denucleated and implanted FEMs for simulation and validation of the experimental data.
The polymeric hydrogel device was modeled by "implanting" the nucleus replacement in the partially denucleated FEMs. The elastomeric hydrogel implant was modeled with a first-order Mooney-Rivlin strain energy potential function, which is a simplified version of Eq. (1):

$$
U=C_{10}\left(\bar{I}_{1}-3\right)+C_{01}\left(\bar{I}_{2}-3\right)+\frac{1}{D_{1}}\left(J_{\mathrm{el}}-1\right)^{2}
$$

The elastic moduli and Poisson's ratios were converted to Mooney-Rivlin constants using the shear modulus and bulk modulus equations, along with the assumption that, in general, for a hyperelastic material with $N=1$ such as for Mooney-Rivlin, $C_{10}$ is four times that of $C_{01}$. The MooneyRivlin coefficients were calculated for various implant moduli, and then used for the simulation of hydrogel implants in the implanted disc models.

Based on the actual dimensions of the test specimens, six individual FEMs were constructed. All six specimens were free of any significant bone or disc abnormalities. The geometric details of each specimen are given in Table II. The material property definitions used in the model for cortical bone, cancellous bone, and nucleus pulposus are given in Table III. Simplified geometry was used for the FEMs, assuming symmetry about the sagittal plane. Contribution of the end plates was neglected. The height of the intervertebral disc was assumed to be uniform over the entire cross-sectional area. Mesh refinement studies were performed. The FEM used 2327 nodes and 1728 four-node axisymmetric elements (Figure 1). The loading simulated the test condition in which the bottom vertebra was constrained in the test fixture using a potting material. A fixed displacement of $15 \%$ strain (based on the disc height) was applied to the top vertebra and symmetric boundary condi-

\begin{tabular}{|c|c|c|c|c|c|c|c|}
\hline & Age & Sex & Total Height & Disc Height & Upper Vertebra & Lower Vertebra & Major Diameter \\
\hline Specimen 1 & 49 & M & 40.0 & 10.0 & 15.0 & 15.0 & 48.0 \\
\hline Specimen 2 & 67 & $\mathrm{~F}$ & 38.0 & 9.5 & 10.5 & 17.5 & 38.0 \\
\hline Specimen 3 & 74 & M & 31.0 & 9.0 & 8.0 & 14.0 & 35.0 \\
\hline Specimen 4 & 67 & $\mathrm{~F}$ & 33.0 & 11.0 & 7.0 & 15.0 & 42.0 \\
\hline Specimen 5 & 47 & $\mathrm{~F}$ & 39.0 & 11.0 & 13.0 & 15.0 & 45.0 \\
\hline Specimen 6 & 47 & $\mathrm{~F}$ & 37.5 & 12.0 & 11.0 & 14.5 & 47.0 \\
\hline AVFEM & & & 36.4 & 10.4 & 10.8 & 15.2 & 42.5 \\
\hline
\end{tabular}

TABLE II. Geometrical Data for Upper Vertebra-Intervertebral Disc-Lower Vertebra Specimens (all dimensions in mm) 
TABLE III. Material Properties Used in Finite Element Model

\begin{tabular}{lrc}
\hline & Young's Modulus (MPa) & Poisson's Ratio \\
\hline Cortical bone & 12,000 & 0.3 \\
Cancellous bone & 100 & 0.2 \\
Nucleus pulposus & 1 & 0.4999 \\
\hline
\end{tabular}

tions were used to perform the nonlinear analysis using the commercially available finite element software (ABAQUS 5.4, ABAQUS Inc., Pawtucket, RI, USA).

\section{Model Validation and Average Finite Element Model}

In a separate physical experiment conducted in our laboratory, $^{28}$ three different conditions-intact, partially denucleated, and implanted-were tested in axial compression. Our approach to denucleating the specimen by removing the nucleus material through a hole bored in the superior vertebra avoided any damage to the annulus fibrosus and maintained it intact throughout the testing., ${ }^{3,28}$ The specimens were denucleated up to a volume ratio of the nuclear cavity to the total nucleus of $80 \%$, as measured after testing and removal of the device. This was simulated in the model by prescribing the outer $20 \%$ of the nucleus, radially, as nucleus pulposus, while the more centrally located region of the nucleus was considered "implanted" from vertebra to vertebra and assigned the modulus of the
(A)

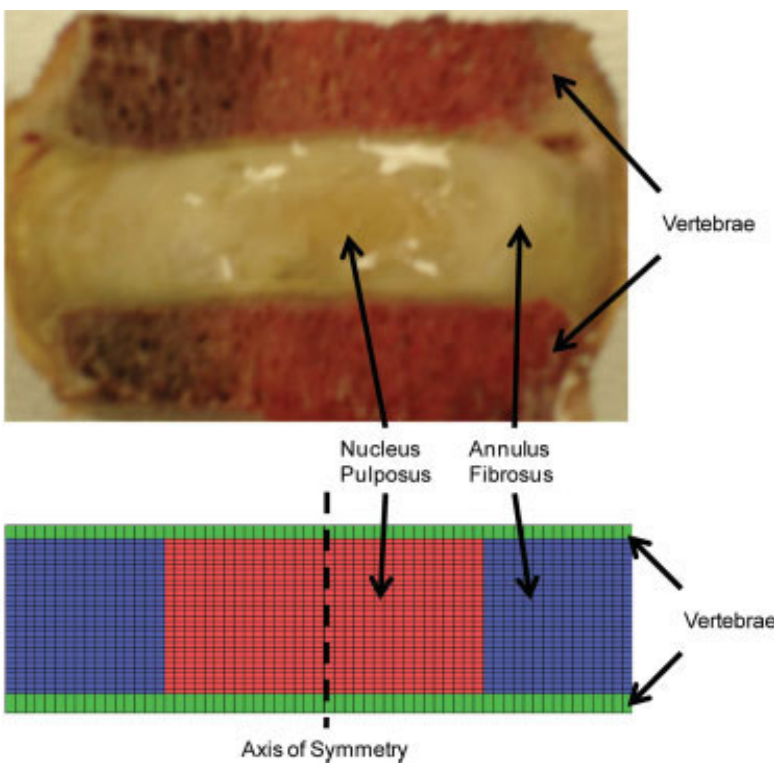

Figure 1. (A) Coronal cross-sectional view of the human intervertebral disc showing the nucleus pulposus, annulus fibrosus, and surrounding vertebrae. (B) Axisymmetric finite element model of the intervertebral disc. [Color figure can be viewed in the online issue, which is available at www.interscience.wiley.com.]

PVA/PVP hydrogel. These different conditions (intact, partially denucleated, and implanted) were simulated for each specimen and then compared to the corresponding experimental results for compressive stiffness.
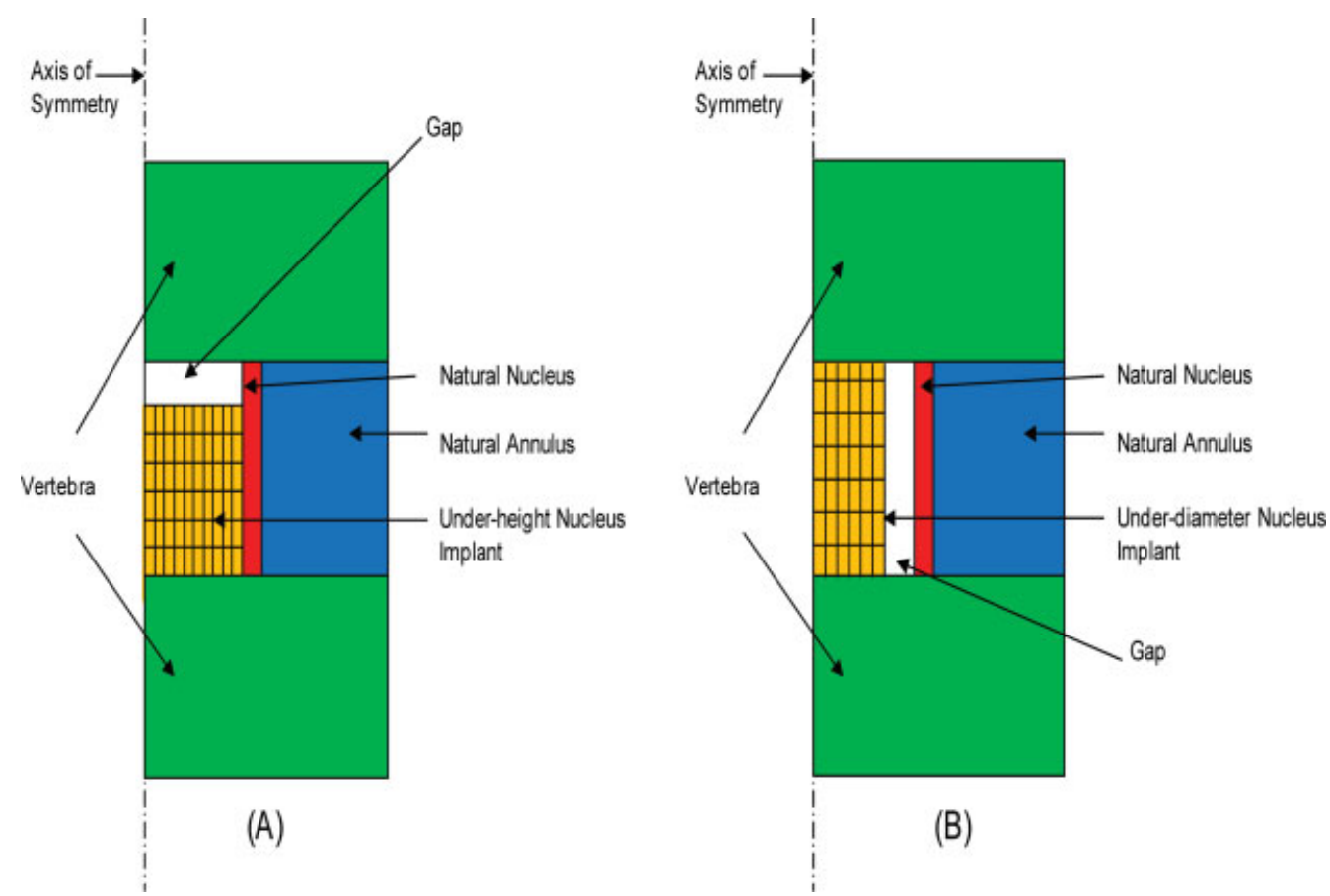

Figure 2. Model design showing the under-filled conditions. (A) Under-height model utilizes a gap between the implant and the proximal vertebral body with a line-to-line fit at the implant-nucleus interface. (B) Under-diameter model leaves a gap between the implant and the remaining nucleus with a line-to-line fit with the proximal and distal vertebrae. [Color figure can be viewed in the online issue, which is available at www.interscience.wiley.com.] 
The test specimens had variations with respect to age, gender, and degenerative state (Table II). The average age of the specimens was 58.5 years (range, 47-74 years); four specimens were from females, two from males; and the degenerative conditions were consistent with age. An average FEM (AVFEM) was built based on the average dimensions of the six specimens. This AVFEM was analyzed with the same loading and boundary conditions as those for the individual FEMs. The annulus parameters used for this AVFEM were taken as the average of the corresponding six annulus parameters determined for individual FEMs (Table I).

As before, the nucleus was removed from the AVFEM to simulate the experimental denucleated condition. The hydrogel implant was then modeled in the denucleated AVFEM to simulate the implanted condition. The required modulus of the hydrogel implant for complete restoration of the intact AVFEM load level, having line-to-line fit, was determined based on the resultant load-displacement behavior.

Using this validated, implanted AVFEM, an analysis of compressive load level, intradiscal stress distribution, radial displacement of peripheral nucleus and annulus layers, compressive stresses on nucleus and annulus, interfacial stresses between the peripheral nucleus and inner annulus, and radial strains in the nucleus and annulus was performed.

\section{Parametric Analysis}

Using a validated representative model in lieu of the AVFEM, the modulus of the nucleus implant was varied from 0.01 to $100 \mathrm{MPa}$ in the implanted condition (with constant Poisson's ratio of 0.4999) to assess the effect of implant modulus on the compressive mechanics of the lumbar intervertebral disc. The nucleus implant was simulated with "line-to-line fit" of the nuclear cavity. The effect of Poisson's ratio of the nucleus implant on the mechanical behavior of the implanted disc was also studied by varying it while maintaining a Young's modulus of $150 \mathrm{kPa}$ and "line-to-line" fit of the nuclear cavity.

Under-filling of the nuclear cavity was simulated by defining the nucleus implant with 3, 5, and $10 \%$ under-fill, either with the height or with the diameter, keeping all other parameters constant (Figure 2). For example, for an implant with 5\% under-height (UH) condition, the modulus, the Poisson's ratio, and the diameter were kept unchanged as in the case of "line-to-line fit," and for an implant with $5 \%$ under-diameter (UD) condition, the modulus, the Poisson's ratio, and the height were kept unchanged. Likewise, an over-fill of the nuclear cavity was simulated by defining the nucleus implant with 3,5 , and $10 \%$ over-fill, either for the height or for the diameter. For the UH and over-height $(\mathrm{OH})$ conditions, the implant maintained a line-to-line fit with the distal vertebral body as well as the nucleus pulposus, while the implant height was altered. Similarly, in the UD and over-diameter (OD) conditions, the implant main-

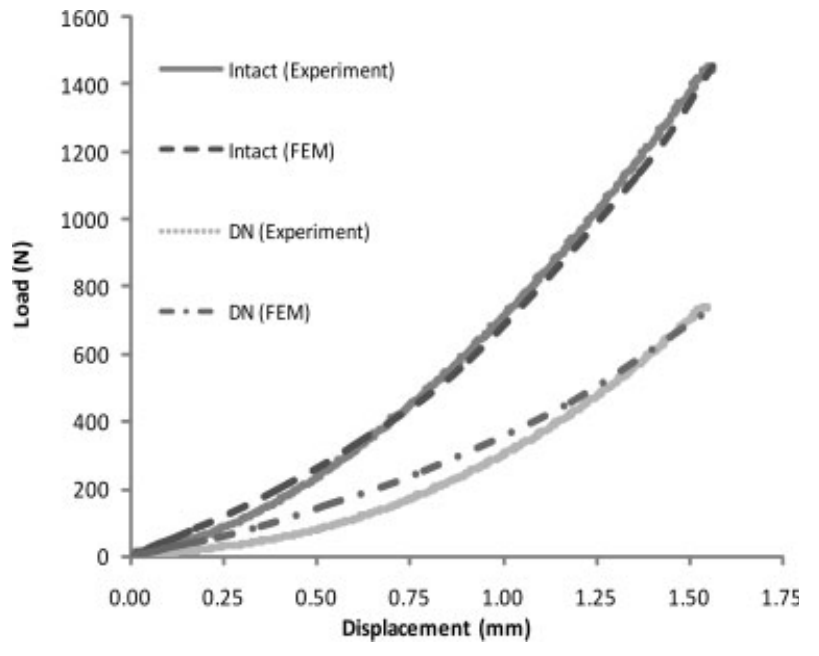

Figure 3. Comparison of intact and denucleated (DN) specimens using experimental and computational data, where the experimental and simulated predictions match well for both intact and denucleated conditions.

tained a line-to-line fit with both the proximal and the distal vertebral bodies, while the diameter was altered.

A contact definition was used in the analyses where the nucleus implant was defined as the slave surface and the surrounding disc material as the master. ${ }^{45}$ This definition prohibited the penetration of the nucleus implant into the disc material. The under-fill cavity analysis was performed in a single step. However, for simulation of the over-fill condition, a two-step analysis was performed. During the first step, the press-fitted implant exhibits a pre-load on the bounds of the nuclear cavity putting stress on the inner annular layers. During the press-fit process, the implant displaces the annulus radially outwards, adding some preload to the disc. In the second step, normal compression occurs, starting with this preloaded condition.

\section{RESULTS}

Figure 3 shows a comparison of the FEM prediction and the experimental cadaveric results, for both intact and denucleated conditions, for a representative specimen, in terms of the load-displacement behavior. The individual predictions from the FEMs matched well with the experimental data for both conditions of intact and denucleated specimens, for all six specimens. Figure 4 shows the AVFEM load-displacement prediction compared to the experimental data for each of the six specimens, for the intact and denucleated conditions. The AVFEM model was able to simulate the nonlinear load-displacement behavior as seen in experimental conditions. The denucleated experimental conditions vary significantly among the species. The denucleated AVFEM model was also able to reproduce the denucleated load-displacement behavior within the range of the experimental findings. 

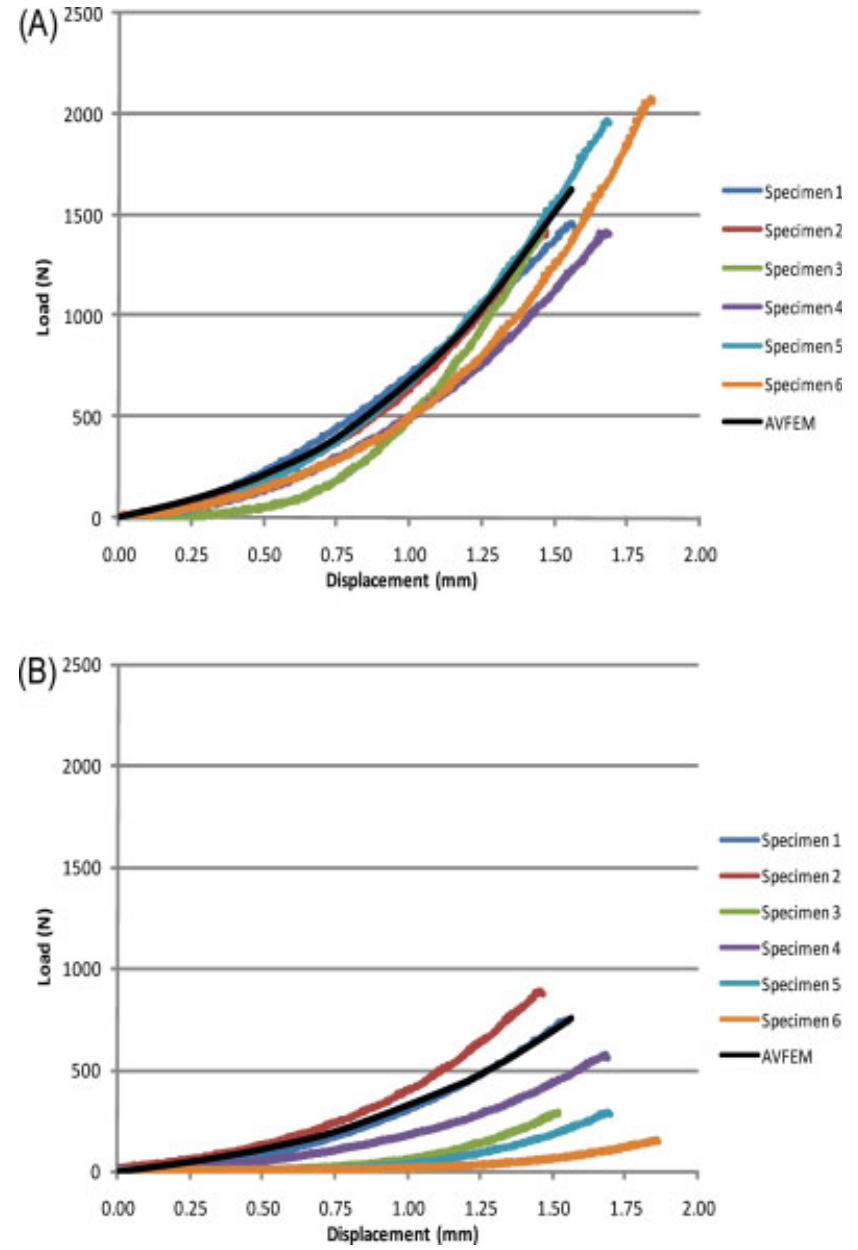

Figure 4. FEM prediction compared to experimental data of $(A)$ intact and (B) denucleated specimens, showing a range of mechanical properties with the AVFEM response lying within this range. The denucleated samples are less stiff than the intact and show a wider variation in load-displacement behavior. [Color figure can be viewed in the online issue, which is available at www.interscience.wiley.com.]

Figure 5 shows the intact, denucleated, and implanted AVFEMs contour comparison for von Mises stress distribution. The intact and denucleated conditions show distinctly different stress profiles in the annulus, while the implanted condition is more comparable to the intact condition for the stress distribution in the annulus. It is interesting to see the different stress distributions in the implanted material (more uniform and higher stress magnitude) compared to the natural nucleus.

The radial strain was analyzed from the axis of symmetry of the model (center of the nucleus) to the outer annulus (Figure 6). The intact and implanted conditions each show a positive radial strain (about 9\%). The denucleated condition, however, shows a compressive strain about 40$60 \%$ of the distance from the axis of symmetry where the remaining nucleus and inner annulus show inward bulging. Through the annulus, the strain then transitions to a tensile state, however the magnitude of the strain never reaches the level of the intact or implanted conditions.
The effect of implant modulus and Poisson's ratio using the AVFEM is shown in Figure 7. There was a moderate effect of implant modulus on the load-displacement behavior, where for a given load (e.g., $1000 \mathrm{~N})$, as the modulus increased, the displacement was reduced. Also in Figure 7, the effect of Poisson's ratio with a constant modulus (150 $\mathrm{kPa}$ ) is seen. There is a moderate change in load-displacement behavior from 0.4999 to 0.45 , however, below 0.45 there are much less dramatic changes in compressive mechanical behavior.

Figure 8 shows the effect of under-filling and over-filling of the nuclear cavity with variation in implant height and diameter. The FEM predictions of the load-displacement behavior for the 3,5, and $10 \% \mathrm{UH}$ (and UD) and $\mathrm{OH}$ (and OD) implants are compared against the intact experimental result (which is equivalent to the line-to-line fit

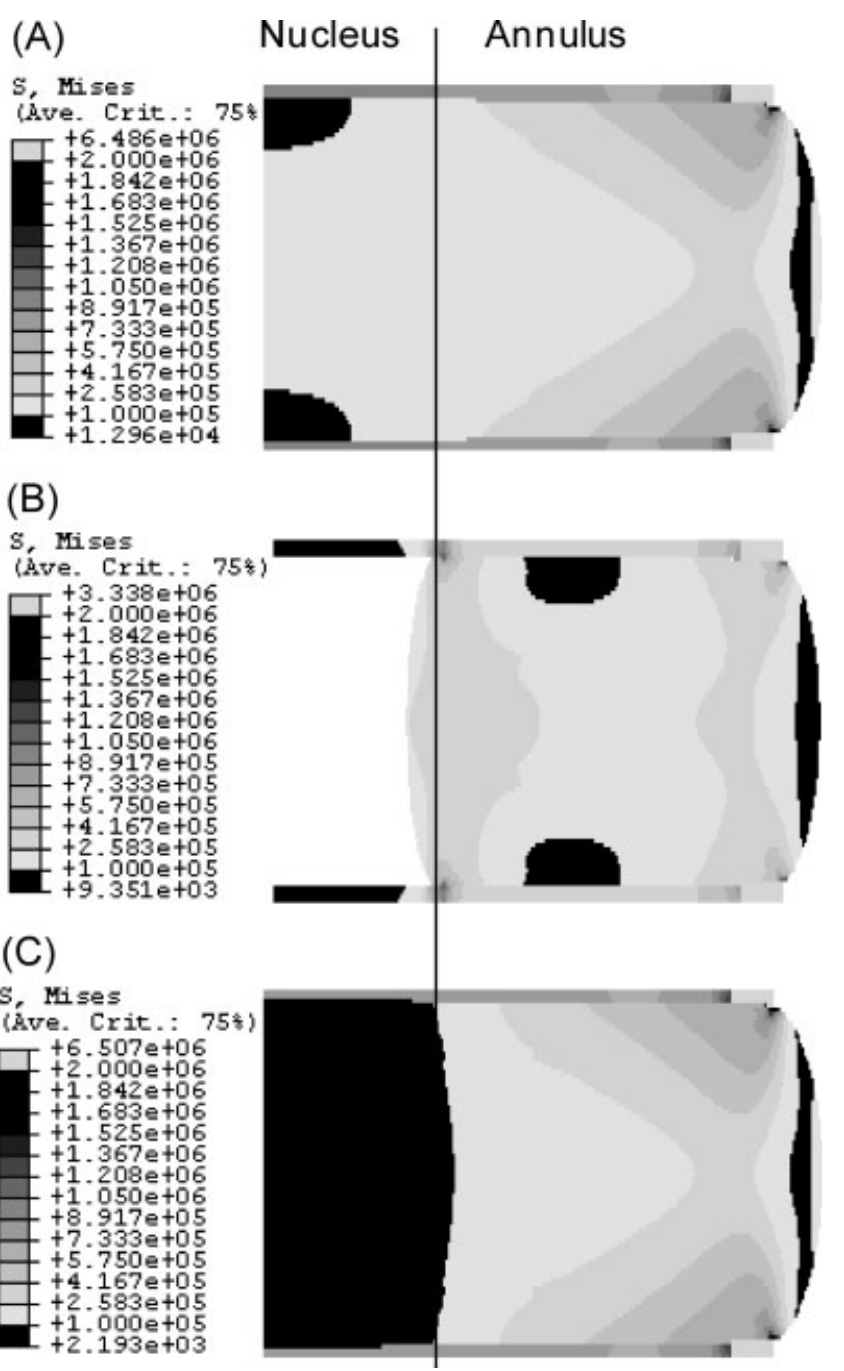

Figure 5. Von Mises stress contours for (A) intact model, (B) denucleated, and (C) implanted. As compared to the intact model, the denucleated response shows an altered stress state as well as an inward bulging while the implanted results restore the stress state in the annulus to the intact condition with a uniform stress in the nucleus replacement. 


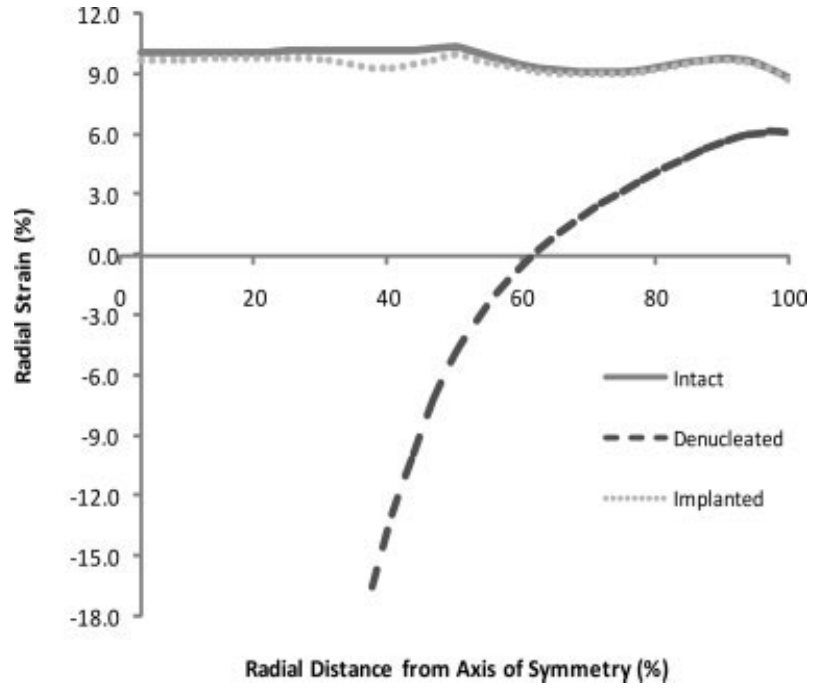

Figure 6. Radial strain distribution of FEM for intact, denucleated, and implanted specimens, with respect to the axis of symmetry. The denucleated curve shows the inward bulging of the annulus in the absence of the nucleus, but the radial strain profile is restored after implantation of the nucleus replacement.

implanted condition). The UH condition shows a more pronounced difference from that of the intact than the $\mathrm{OH}$ does at higher loads $(>400 \mathrm{~N})$, while at lower loads $(<400$ $\mathrm{N})$, the UH is closer to the intact. Likewise, the UD curves are nearer to the intact at lower loads and more different than the ODs at higher loads.

Von Mises stress distribution for different implanted conditions is compared in Figure 9 (10\% UH and 10\% OH, $10 \%$ UD and $10 \%$ OD) with the stress distribution for the line-to-line fit implant. Figure 10 compares the radial displacement contours for different implanted conditions with the line-to-line fit contour. It was observed that with the under-filled implant (either height or diameter), the annulus showed less radial displacement compared to the line-toline fit implant. As a result, the restoration load level was less for the under-filled condition.

\section{DISCUSSION}

Nucleus pulposus replacements are quickly emerging as treatments for early degeneration of the lumbar intervertebral disc. ${ }^{9-11,46}$ Little, however, is understood about the relationship between the nucleus implant properties and mechanical behavior of the implanted disc. This work presents a simplified FEM to parametrically examine the relationship between implant properties and compressive mechanics of the intervertebral disc.

\section{Modeling Approach}

We have modeled the polymeric nucleus implant using Mooney-Rivlin strain energy potential and the annulus fibrosus as an isotropic, hyperelastic material with second- order polynomial strain energy function, in agreement with the strategy put forth by Duncan and Lotz. ${ }^{34}$ This strategy was used on six separate discs to create six separate FEMs. The properties of the discs were then averaged to form a representative AVFEM model, which may be more representative of the range of disc properties seen experimentally. ${ }^{17,28,36}$ The models were validated against experimental load-displacement behavior.

\section{Effect of Denucleation on Intervertebral Disc Mechanics}

The compressive load-displacement curves of the intact disc showed characteristic nonlinearity, with a clearly defined neutral zone, as is seen experimentally in axial compression. ${ }^{47}$ There was a positive von Mises stress on the intact specimen where the nucleus and annulus meet (Figure 5), as well as a positive radial strain for the intact condition across the specimen (Figure 6), with an outward bulging of the inner as well as outer annulus. This indicates
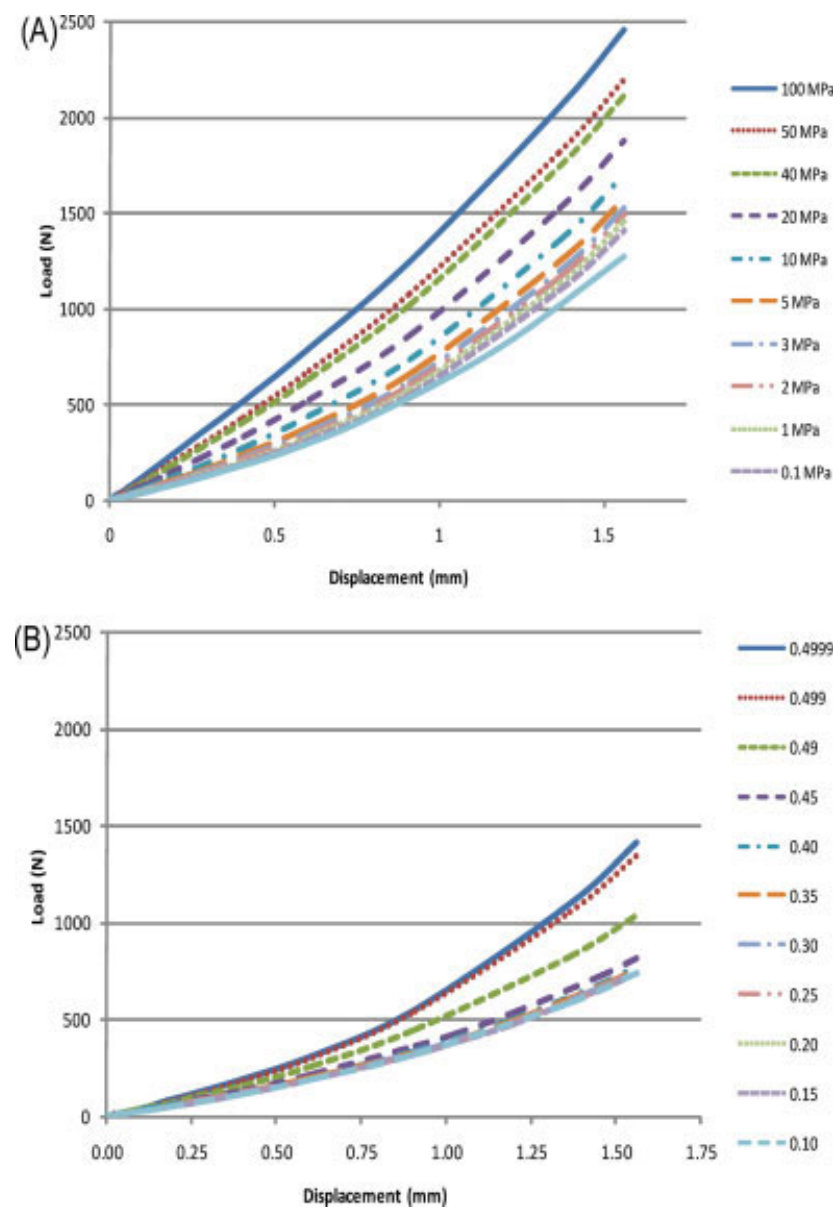

Figure 7. Load-displacement curves for various (A) elastic moduli and (B) Poisson's ratios. The implant modulus curves show that, for a given load, as the modulus increases, the displacement decreases. Compressive behavior varies greatly for Poisson's ratios in the range of $0.45-0.4999$, but shows little change below 0.45 . [Color figure can be viewed in the online issue, which is available at www.interscience.wiley.com.] 

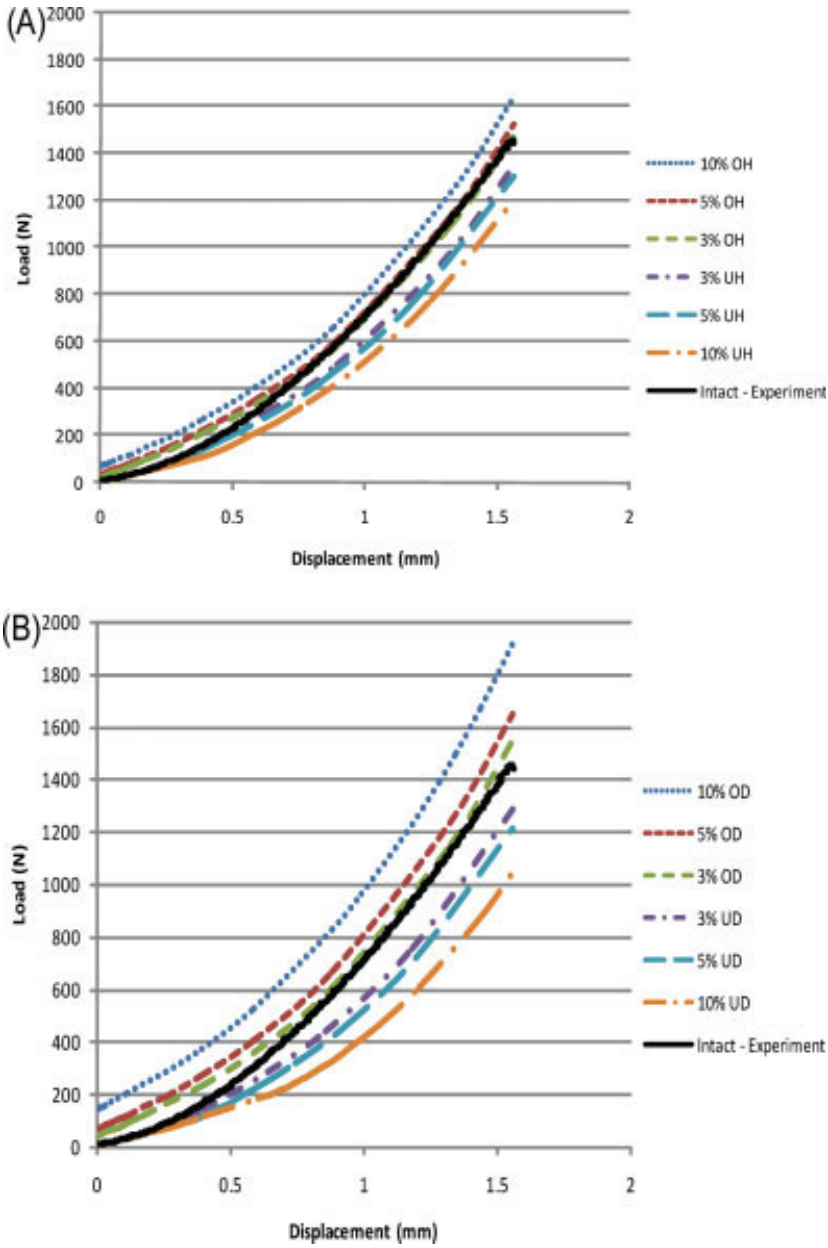

Figure 8. Load-displacement curve for various (A) implant heights and (B) implant diameters. The over-height/diameter conditions are more representative of intact than the under-heights/diameters at higher loads, while the reverse is true at lower loads $(<400 \mathrm{~N})$. [Color figure can be viewed in the online issue, which is available at www.interscience.wiley.com.]

that under normal compressive conditions, the nucleus is able to tense the annulus. This can be compared, however, to the denucleated condition. Here, there are drastically different stress patterns in the annulus with an inward bulging of the inner annulus, while maintaining the outward bulging of the outer annulus (Figure 5). The intradiscal stress, the stress acting between the nucleus and the annulus reduced $30 \%$ to that of the intact condition. The strains in the inner annulus are compressive and only transition over to tensile strains in the mid-thickness region of the annulus (Figure 6). These findings are in keeping with those of Meakin et al., ${ }^{41}$ who showed the inner annulus bulging inward in a compressive buckling behavior under denucleated conditions both experimentally and using numerical modeling. Our group has also shown that the compressive mechanics are dependent on the degree of denucleation, experimentally. ${ }^{15}$ Increased removal of the nucleus shows more instability of the disc, especially at low load levels as measured by neutral zone mechanics and stiffness in the neutral zone.

\section{Effect of Implant Modulus and Poisson's Ratio on Compressive Mechanics}

We modeled the effect of a nucleus implant on compressive biomechanics of the disc by "implanting" a nucleus implant with a modulus of $150 \mathrm{kPa}$ and a Poisson's ratio of 0.499 using the Mooney-Rivlin method described above, with a line-to-line fit. It can be clearly seen that the effect of implantation can restore the denucleated condition close to the original intact condition. The stress distribution in the annulus is more like the intact and the tensile stresses are restored to the entire radial profile. The implanted disc still maintains its nonlinear load-displacement behavior.

For the implanted AVFEM, the modulus of the hydrogel nucleus implant was varied to understand the effect of implant modulus on compressive biomechanics. When comparing the load-displacement curves for the discs implanted with different moduli, there were only small variations in compressive behavior for implants with a modulus over three orders of magnitude, from 0.01 to $5.00 \mathrm{MPa}$. These results are consistent with experimental work showing that each implant in a modulus range from 50 to 1500 $\mathrm{kPa}$ restored denucleated mechanics back to the intact levels, but showed no statistical differences between implants of the different moduli in the range tested. ${ }^{28}$ However, between 5 and $100 \mathrm{MPa}$, there were more dramatic changes in the compressive behavior with increased stiffness of the disc. At the higher levels of implant modulus, the nucleus no longer acts to pressurize the annulus to put it into tension, instead, the nucleus becomes a rigid column support and shields the annulus from stress. This transition occurs when the modulus of the nucleus implant exceeds the modulus of the annulus, around $5 \mathrm{MPa}$. Different nucleus replacement designs work in different regions of this spectrum. Some aim to distract the disc by providing a more rigid columnar support in replacement of the nucleus, while others are more biomimetic, aiming to reproduce normal intradiscal stresses, through the incorporation of an elastomeric, low modulus material. Differences in patient outcome (pain relief) remain to be seen at this point in time. It is important to consider the stress that a high modulus nucleus implant will exert on the endplates and to limit this stress to physiologically relevant levels, so as not to cause endplate subsidence.

We also examined the effect of the Poisson's ratio of the nucleus replacement on the compressive behavior of the disc using a constant $150 \mathrm{kPa}$ implant modulus. Between a Poisson's ratio of 0.1 and 0.45 there was little variation in the load-displacement behavior. In this range, there was an inner bulging of the inner annulus, indicating that the implant was not able to provide a tensile loading to the annulus and showing that the implant with these Poisson's ratios was not able to increase disc stiffness. 


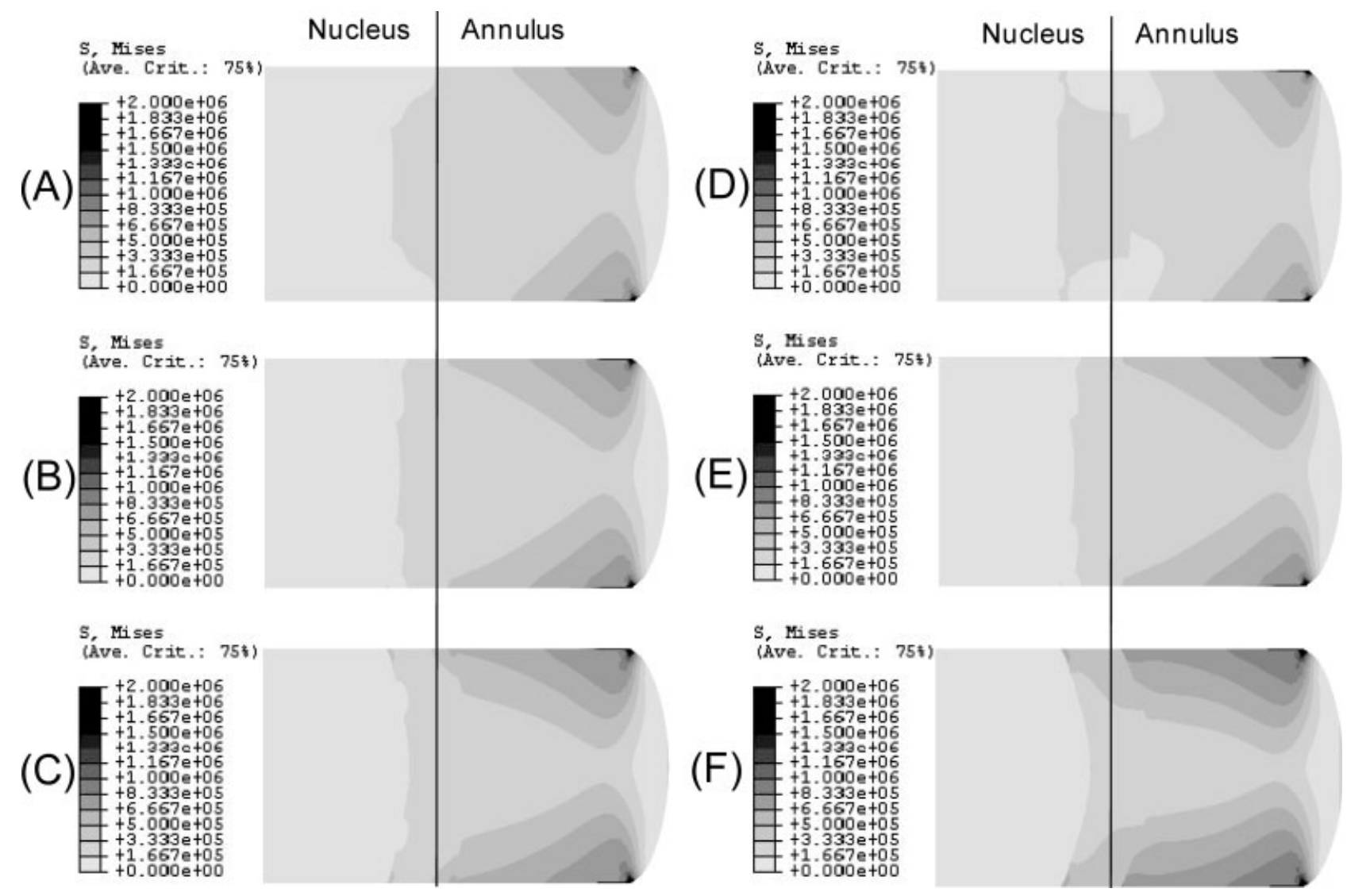

Figure 9. Von Mises stress distributions for (A) 10\% under-height implant, (B) line-to-line fit, (C) $10 \%$ over-height, (D) 10\% under-diameter, (E) line-to-line fit, (F) 10\% over-diameter ranging from 0 to $2.0 \mathrm{MPa}$.

(A)
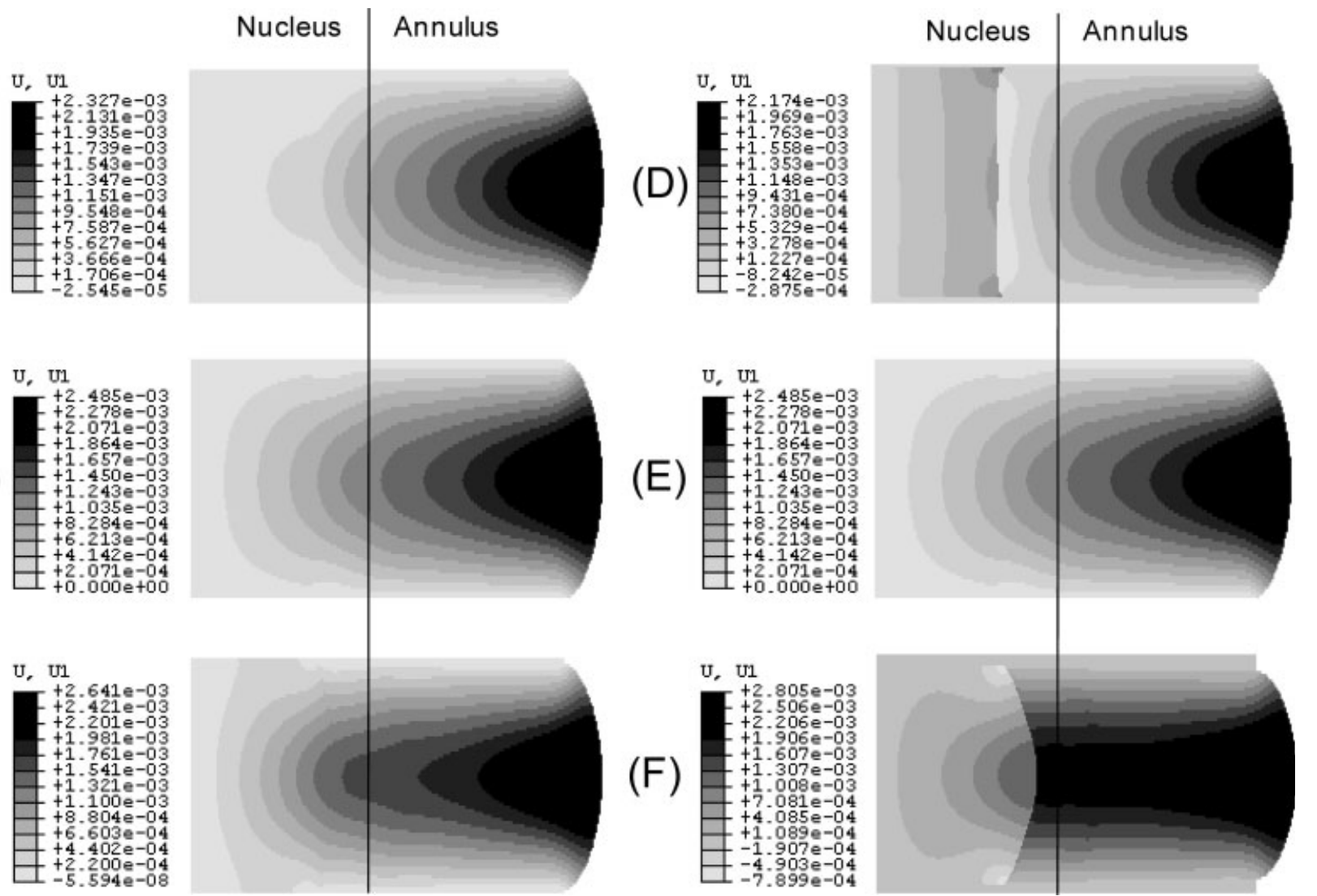

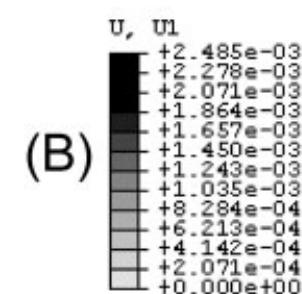
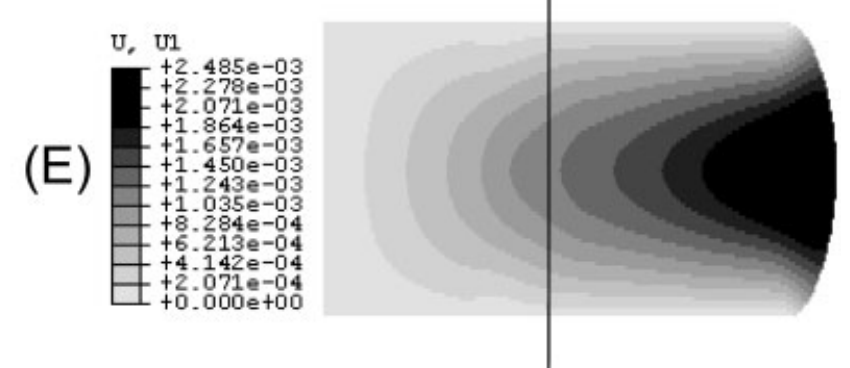

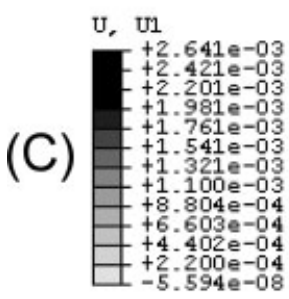

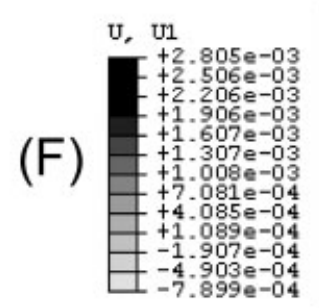

Figure 10. Displacement distributions for (A) 10\% under-height implant, (B) line-to-line fit, (C) $10 \%$ over-height, (D) 10\% under-diameter, $(E)$ line-to-line fit, $(F) 10 \%$ over-diameter ranging from 0 to $2.5 \mathrm{~mm}$. 
However, in the more elastomeric regions between 0.45 and 0.499 , there were more dramatic differences, with higher stiffness occurring with higher nucleus implant Poisson's ratio. Moreover, the predicted behavior with lower values of implant Poisson ratios $(v \leq 0.45)$ was comparable to the predicted behavior of the denucleated disc. This indicates the importance of the incompressibility of the implanted material and suggests the use of implant materials with higher Poisson's ratio. With the relatively low modulus used in this analysis, the degree of radial deformation to engage the annulus becomes important in the tensioning of the annulus fibrosus and functioning of the implanted disc.

\section{Fit and Fill Parametric Analysis}

We have modeled the effect of under-filling and over-filling a nuclear cavity on the compressive biomechanics for the first time. Just as Poisson's ratio affects the radial stress applied from the nucleus to the annulus, so does the degree of filling for biomimetic implants in the low modulus regime, where the intradiscal pressure is being mimicked with an intradiscal radial stress. Both $\mathrm{OH}$ and OD implants showed increased stiffness of the load-displacement curves. The over-filled diameter implants showed more dramatic differences from the line-to-line models than the over-filled heights. Primarily this is a volume effect and the diameter squared would have more of an impact than the height of a cylindrical nucleus replacement.

The under-filled cavities needed more deformation to reach the same load levels as the line-to-line or over-filled conditions. For the low load levels $(<400 \mathrm{~N})$, the under filled implants had a less dramatic change in the loaddeformation curve than the over filled implants. Once the higher levels of loading were achieved, the differences between under-filling and line-to-line fit were more dramatic. While the over-filled and line-to-line implants ended up with the same slope, the under-filled implants never achieved this level of disc stiffness. The under-filled implants were not as effective at restoring loads to the intact condition as the over-filled or line-to-line. These effects are clearly visualized in Figure 9, where the overfilled implants have a stress profile that clearly prestresses the annulus.

Clear differences were also seen in the radial displacement (Figure 10) distributions among the under-filled, lineto-line, and over-filled conditions. The implants in the under-filled conditions have a much lower and more columnar displacement profile than the line-to-line or over-filled, which show higher implant stresses, corresponding with more highly tensile annular displacements. These stress distribution lead to earlier tensioning of the annulus fiber and in the case of over-filled implants, not only restoration to intact levels, but the ability to increase the stiffness of the disc to higher levels, controlled by the degree of interfer- ence of the nucleus replacement device with the surrounding tissue.

The slave/master approach for modeling the boundaries between over-filled implants and the surrounding tissue provides a new approach in nucleus replacement analysis of fit and fill. This approach was robust up to about $13 \%$ interference, which was the limit of the technique. We report values well within that limit ( $10 \%$ over-filled). The current model does not take into account other loading conditions, such as anterior/posterior bending, extension, and torsion. In that case, the anisotropy of the annulus may become more dominant and may demand the material definition of the annulus to best represent the in vitro experimental conditions. In addition, more complex geometry, modeled in 3D would also benefit alternate loading conditions where the asymmetry of the geometry will play a more dominant role in the mechanical behavior of the disc.

The axisymmetric geometry with nonlinear material properties used to model the lumbar intervertebral disc and nucleus replacement devices provided valuable information for our parametric assessment of the effect of implant modulus and Poisson's ratio as well as fit and fill of the device in the nuclear cavity on the compressive biomechanics of the intact, denucleated, and implanted disc. We have shown that within a low modulus regime, the stiffness of the disc and stress profiles are highly dependent on volumetric filling, and less dependent on implant modulus. While for higher modulus devices, a different stress state (one of stress shielding, rather than load sharing) is achieved and the higher the modulus, the higher the stiffness of the disc. This work shows that the nucleus replacements have the ability to alter the stress state of the annulus fibrosus and to restore compressive stiffness of the disc to intact levels.

\section{CONCLUSIONS}

It was concluded that the nucleus implant with suitable material properties and dimensions exerts the stress on the inner annulus layers, equivalent to the natural intradiscal pressure in the case of healthy disc. This implantation of the nucleus implant mimics the natural load transfer phenomenon of the healthy disc, by pushing the annulus radially outwards. Nucleus implant design may be bettered by consideration of the implant material/geometric parameters and relative implant volume to the cavity volume.

The authors thank Dr. Antonios Zavaliangos for technical guidance.

\section{REFERENCES}

1. Chan M, Chowchuen P, Workman T, Eilenberg S, Schweitzer M, Resnick D. Silicone synovitis: MR imaging in five patients. Skeletal Radiol 1998;27:13-17.

2. Gan J, Ducheyne P, Vresilovic E, Shapiro I. Bioactive glass serves as a substrate for maintenance of phenotype of nucleus 
pulposus cells of the intervertebral disc. J Biomed Mater Res 2000;51:596-604.

3. Joshi A, Fussell G, Thomas J, Hsuan A, Lowman A, Karduna A, Vresilovic E, Marcolongo M. Functional compressive mechanics of a PVA/PVP nucleus pulposus replacement. Biomaterials 2006;27:176-184.

4. Hamilton D, Seguin C, Wang J, Pilliar R, Kandel R. Formation of a nucleus pulposus-cartilage endplate construct in vitro. Biomaterials 2006;27:397-405.

5. Mizuno H, Roy A, Zaporojan V, Vacanti C, Ueda M, Bonassar L. Biomechanical and biochemical characterization of composite tissue-engineered intervertebral disc. Biomaterials 2006;27:362-370.

6. Sakai D, Mochida J, Iwashina T, Hiyama A, Omi H, Imai M, Nakai T, Ando K, Hotta T. Regenerative effects of transplanting mesenchymal stem cells embedded in atelocollagen to the degenerated intervertebral disc. Biomaterials 2006;27:335345.

7. Sakai D, Mochida J, Yamamoto Y, Nomura T, Okuma M, Nishimura K, Nakai T, Ando K, Hotta T. Transplantation of mesenchymal stem cells embedded in Atelocollagen gel to the intervertebral disc: A potential therapeutic model for disc degeneration. Biomaterials 2003;24:3531-3541.

8. Risbud M, Albert T, Guttapalli A, Vresilovic E, Hillibrand A, Vaccaro A, Shapiro I. Differentiation of mesenchymal stem cells towards a nucleus pulposus-like phenotype in vitro: Implications for cell-based transplantation therapy. Spine 2004;29:2627-2632.

9. Jin D, Qu D, Zhao L, Chen J, Jiang J. Prosthetic disc nucleus (PDN) replacement for lumbar dics herniation. J Spinal Disord Tech $2003 ; 16: 331-337$.

10. Sherman J, Bowman B, Ahrens M, Halm H, Huec JL, Liljenqvist U, Donkersloot P, Hook S, Yuan H. Functional lumbar artificial nucleus replacement-DASCOR. In: Szpalski M, Gunzburg R, Huec JL, Brayda-Bruno M, editors. Nonfusion Technologies in Spine Surgery. Philadelphia, PA: Lippincott, William, \& WIlkins; 2006. p 131.

11. Shim C, Lee S, Park C, Choi W, Choi G, Choi W, Lim S, Lee H. Partial disc replacement with the PDN prosthetic disc nucleus device. J Spinal Disord Tech 2003;16:324-330.

12. Weber H. Lumbar disc herniation: A controlled, prospective study with ten years of observation. Spine 1983;8: $131-140$.

13. Kambin P, Savitz M. Arthroscopic microdiscectomy: An alternative to open disc surgery. Mt Sinai J Med 2000;67:283287.

14. Blumenthal S, McAfee P, Guyer R, Hochschuler S, Geisler F, Holt R, Garcia R, Regan J, Ohnmeiss D. A prospective, randomized, multicenter Food and Drug Administration investigational device exemptions study of lumbar total disc replacement with the Charite artificial disc versus lumbar fusion. Spine 2005;30:1565-1575.

15. Cannella M, Arthur A, Allen S, Joshi A, Vresilovic E, Marcolongo M. The role of the nucleus pulposus in neutral zone human lumbar intervertebral disc mechanics. J Biomech 2008; 41:2104-2111.

16. Bao Q, McCullen G, Higham P, Dumbleton J, Yuan H. The artificial disc: Theory, design and materials. Biomaterials 1996; 17:1157-1166.

17. White A, Panjabi M. Clinical Biomechanics of the Spine. Philadelphia: J.B. Lippincott Company; 1990.

18. Tropiano P, Huang R, Girardi F, Cammisa F, Marnay T. Lumbar total disc replacement: Seven to eleven year followup. J Bone Joint Surg Am 2005;87:490-496.

19. Luoma K, Riihimaki H, Luukkonen R, Raininko R, ViikariJuntura E, Lamminen A. Low back pain in relation to lumbar disc degeneration. Spine 2000;25:487-492.
20. Olczyk K. Age-related changes in glycosaminoglycans of human intervertebral discs. Folia Histochem Cytobiol 1993; 31:215-220.

21. Olczyk K. Age-related changes in proteoglycans of human intervertebral discs. Z Rheumatol 1994;53:19-25.

22. Roughley P, Alini M, Antoniou J. The role of proteoglycans in aging, degeneration and repair of the intervertebral disc. Biochem Soc Trans 2002;30:869-874.

23. Urban J, McMullin J. Swelling pressure of the lumbar intervertebral discs: Influence of age, spinal level, composition, and degeneration. Spine 1988;13:179-187.

24. Urban J, Roberts S. Degeneration of the intervertebral disc: Review. Arthritis Res Ther 2003;5:120-130.

25. Yerramalli C, Chou A, Miller G, Nicoll S, Chin K, Elliott D. The effect of nucleus pulposus crosslinking and glycosaminoglycan degradation on disc mechanical function. Biomech Model Mechanobiol 2007;6:13-20.

26. Guerin H, Elliott D. Degeneration affects the fiber reorientation of human annulus fibrosus under tensile load. J Biomech 2006;39:1410-1418.

27. Fussell G, Thomas J, Scanlon J, Lowman A, Marcolongo $M$. The effect of protein-free versus protein-containing medium on the mechanical properties and uptake of ions of PVA/PVP hydrogels. J Biomater Sci Polym Ed 2005;16: 489-503.

28. Joshi A, Mehta S, Vresilovic E, Karduna A, Marcolongo M. Nucleus implant parameters significantly change the compressive stiffness of the human lumbar intervertebral disc. J Biomech Eng 2005;127:536-540.

29. Thomas J, Gomes K, Lowman A, Marcolongo M. The effect of dehydration history on PVA/PVP hydrogels for nucleus pulposus replacement. J Biomed Mater Res Part B: Appl Biomater 2004;69:135-140.

30. Thomas J, Lowman A, Marcolongo M. Novel associated hydrogels for nucleus pulposus replacement. J Biomed Mater Res Part A 2003;67:1329-1337.

31. Liu X, Marcolongo M, Lowman A. Short term in vitro response of associating hydrogels. 2001. Submitted for publication.

32. Argoubi M, Shirazi-Adl S. Poroelastic creep response analysis of a lumbar motion segment in compression. J Biomech 1996;29:1331-1339.

33. Belytschko T, Kulak R, Schultz A, Galante J. Finite element stress analysis of an intervertebral disc. J Biomech 1974;7:277285.

34. Duncan N, Lotz J. Experimental validation of a porohyperelastic finite element model of the annulus fibrosus. In: Middleton J, Jones M, Pande G, editors. Computer Methods in Biomechanics and Biomedical Engineering-2. London: Gordon and Breach Science Publishers; 1998. pp 527534.

35. Ferguson $\mathrm{S}$, Ito $\mathrm{K}$, Nolte L. Fluid flow and convective transport of solutes within the intervertebral disc. J Biomech 2004;37:213-221.

36. Goel V, Monroe B, Gilbertson L, Brinckmann P. Interlaminar shear stresses and laminae separation in a disc. Finite element analysis of the L3-L4 motion segment subjected to axial compressive loads. Spine 1995;20:689-698.

37. Iatridis J, Laible J, Krag M. Influence of fixed charge density magnitude and distribution on the intervertebral disc: Applications of a poroelastic and chemical electric (PEACE) model. J Biomech Eng 2003;125:12-24.

38. Laible J, Pflaster D, Krag M, Simon B, Haugh L. A poroelastic-swelling finite element model with application to the intervertebral disc. Spine 1993;18:659-670.

39. Shirazi-Adl S, Shrivastava S, Ahmed A. Stress analysis of the lumbar disc-body unit in compression. A three-dimensional nonlinear finite element study. Spine 1984;9:120-134. 
40. Yao J, Turteltaub S, Ducheyne P. A three-dimensional nonlinear finite element analysis of the mechanical behavior of tissue engineered intervertebral disc under complex loads. Biomaterials 2006;27:377-387.

41. Meakin J, Reid J, Hukins D. Replacing the nucleus pulposus of the intervertebral disc. Clin Biomech (Bristol, Avon) 2001;16:560-565.

42. Bao Q, Yuan H. New technologies in spine: Nucleus replacement. Spine 2002;27:1245-1247.

43. Iatridis J, Weidenbaum M, Setton L, Mow V. Is the nucleus pulposus a solid or a fluid? Mechanical behaviors of the nucleus pulposus of the human intervertebral disc. Spine 1996;21:1174-1184.
44. Nagy G, Gentle C. Significance of the annulus properties to finite element modeling of intervertebral discs. J Musculoskeletal Res 2001;5:159-171.

45. ABAQUS. ABAQUS Theory and User's Manual. Providence, RI: Hibbitt, Karlsson and Sorenson, Inc.; 2002.

46. Marcolongo M, Cannella M, Massey C. Nucleus replacement of the intervertebral disc. In: Kurtz S, Edidin A, editors. Spine Technology Handbook. Burlington, MA: Academic Press; 2006. pp 281-302.

47. Gay R, Ilharreborde B, Zhao K, Zhao C, An K. Sagittal plane motion in the human lumbar spine: Comparison of the in vitro quasitatic neutral zone and dynamic motion parameters. Clin Biomech 2006;21:914-919. 